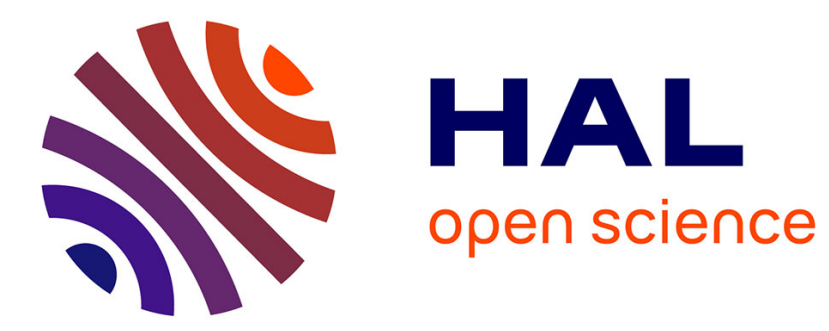

\title{
Low frequency vibration induced streaming in a Hele-Shaw cell
}

\author{
M Costalonga, Philippe Brunet, H Peerhossaini
}

\section{To cite this version:}

M Costalonga, Philippe Brunet, $\mathrm{H}$ Peerhossaini. Low frequency vibration induced streaming in a Hele-Shaw cell. Physics of Fluids, 2015, 10.1063/1.4905031 . hal-01297164

HAL Id: hal-01297164

https: / hal-univ-paris.archives-ouvertes.fr/hal-01297164

Submitted on 4 Apr 2016

HAL is a multi-disciplinary open access archive for the deposit and dissemination of scientific research documents, whether they are published or not. The documents may come from teaching and research institutions in France or abroad, or from public or private research centers.
L'archive ouverte pluridisciplinaire HAL, est destinée au dépôt et à la diffusion de documents scientifiques de niveau recherche, publiés ou non, émanant des établissements d'enseignement et de recherche français ou étrangers, des laboratoires publics ou privés. 


\section{AIP | Physics of}

\section{Low frequency vibration induced streaming in a Hele-Shaw cell}

M. Costalonga, P. Brunet, and H. Peerhossaini

Citation: Physics of Fluids (1994-present) 27, 013101 (2015); doi: 10.1063/1.4905031

View online: http://dx.doi.org/10.1063/1.4905031

View Table of Contents: http://scitation.aip.org/content/aip/journal/pof2/27/1?ver=pdfcov

Published by the AIP Publishing

\section{Articles you may be interested in}

Experimental and numerical study of buoyancy-driven single bubble dynamics in a vertical Hele-

Shaw cell

Phys. Fluids 26, 123303 (2014); 10.1063/1.4903488

Interaction of a synthetic jet with the flow over a low aspect ratio cylinder

Phys. Fluids 25, 064104 (2013); 10.1063/1.4811710

Acoustic radiation by vortex induced flexible wall vibration

J. Acoust. Soc. Am. 118, 2182 (2005); 10.1121/1.2011127

Kelvin-Helmholtz instability in a Hele-Shaw cell

Phys. Fluids 14, 922 (2002); 10.1063/1.1446884

Gravitational instability of miscible fluids in a Hele-Shaw cell

Phys. Fluids 14, 902 (2002); 10.1063/1.1431245

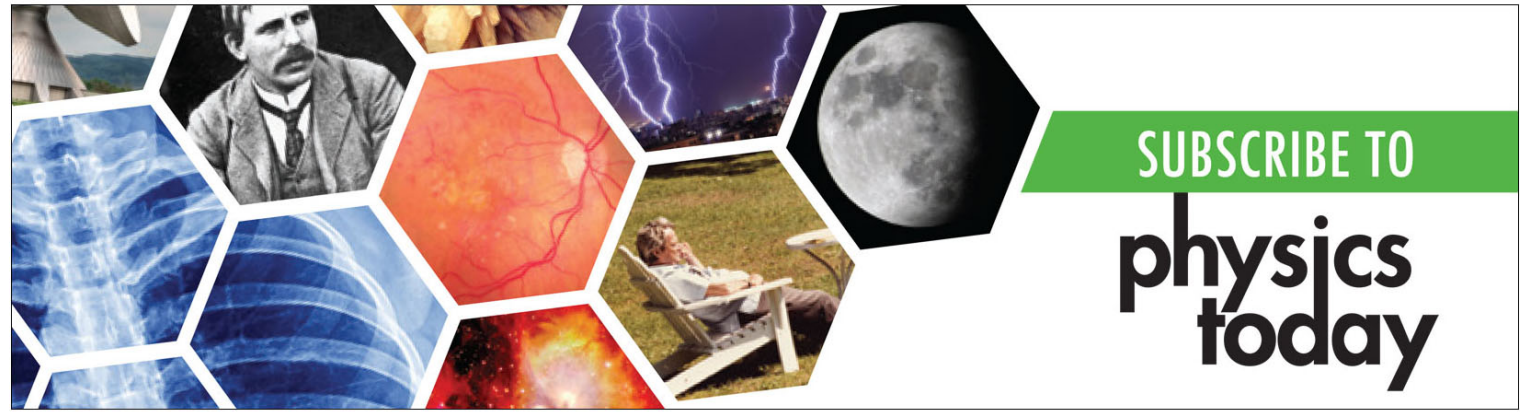




\title{
Low frequency vibration induced streaming in a Hele-Shaw cell
}

\author{
M. Costalonga, ${ }^{1,2, a)}$ P. Brunet, ${ }^{2}$ and H. Peerhossaini ${ }^{1}$ \\ ${ }^{1}$ Laboratoire Interdisciplinaire des Energies de Demain (LIED) - Université Paris Diderot, \\ 10 rue Alice Domon et Léonie Duquet, 75205 Paris cedex 13, France \\ ${ }^{2}$ Laboratoire Matière et Systèmes Complexes, UMR CNRS 7057, Université Paris Diderot, \\ 10 rue Alice Domon et Léonie Duquet, 75205 Paris cedex 13, France
}

(Received 16 May 2014; accepted 10 November 2014; published online 5 January 2015)

\begin{abstract}
When an acoustic wave propagates in a fluid, it can generate a second order flow whose characteristic time is much longer than the period of the wave. Within a range of frequency between ten and several hundred $\mathrm{Hz}$, a relatively simple and versatile way to generate streaming flow is to put a vibrating object in the fluid. The flow develops vortices in the viscous boundary layer located in the vicinity of the source of vibrations, leading in turn to an outer irrotational streaming called Rayleigh streaming. Because the flow originates from non-linear time-irreversible terms of the Navier-Stokes equation, this phenomenon can be used to generate efficient mixing at low Reynolds number, for instance in confined geometries. Here, we report on an experimental study of such streaming flow induced by a vibrating beam in a Hele-Shaw cell of $2 \mathrm{~mm}$ span using long exposure flow visualization and particle-image velocimetry measurements. Our study focuses especially on the effects of forcing frequency and amplitude on flow dynamics. It is shown that some features of this flow can be predicted by simple scaling arguments and that this vibration-induced streaming facilitates the generation of vortices. (C) 2015 AIP Publishing LLC. [http://dx.doi.org/10.1063/1.4905031]
\end{abstract}

\section{INTRODUCTION}

It has been known since Faraday ${ }^{1}$ that a steady flow can emanate from a periodic forcing in a fluid, either mechanical vibrations or acoustic waves. A global feature of these fluid flows is that they originate from time-irreversible convection terms of the Navier-Stokes equation ${ }^{2}$ and are thus attractive in overcoming some of the intrinsic challenges of low-Reynolds-number flows as for active mixing, ${ }^{3}$ pumping, ${ }^{4}$ heat transfer,,${ }^{5}$ or particles sorting. ${ }^{6}$ Such streaming has been recently identified to play a significant role in the mixing process occurring in the acinar region (In the case of lungs, the acinar region corresponds to the groups of alveoli, linked by alveolated ducts, terminating the bronchioles.) of lungs. ${ }^{7}$

Acoustic-induced flows have recently received much attention ${ }^{8}$ due to the massive development of microfluidics. Acoustofluidics phenomena are considered especially promising within $\mathrm{MHz}$ frequency ranges, ${ }^{9}$ where acoustic wavelengths become comparable in size to the channel spans. In these situations, the flow is strongly influenced by the interplay between geometrical confinement and the size and shape of the transducer, and these constitute additional challenges in determining optimized flow conditions. Though various historical studies have focused on situations with much lower forcing frequency, they showed that large-scale flows could be generated within tanks of tens of centimeters size, induced by mechanical vibrations of frequency from 1 to $100 \mathrm{~Hz} \cdot{ }^{10-12}$ In such situations, analytical and numerical study of the flow becomes more tractable. ${ }^{13,14}$

The present study aims to bridge the gap between these two approaches to streaming by analyzing the flow induced by a beam vibrating at low frequency in a cell filled with liquid confined

a) maxime.costalonga@univ-paris-diderot.fr 
on two dimensions (Hele-Shaw cell). We aim to demonstrate that confinement makes our results relevant and universal enough for a comparative understanding of phenomena occurring within $\mathrm{MHz}$ acoustic forcing in microfluidics.

The paper is organized as follows: first, a summary description on vibration-induced streaming is presented in Sec. II. The experimental setup is described in Sec. III; then, in Sec. IV, we present the flow pattern qualitatively and show quantitative results based on velocity-field measurements. Finally, Sec. IV proposes theoretical considerations and Sec. V discusses the model and remaining open issues and concludes by describing possible future work.

\section{THEORETICAL GROUNDS}

Vibration-induced streaming is a mean flow that arises from Reynolds stresses under wave propagation ${ }^{15}$ and compressibility. That is, vibration-induced streaming is a second order effect that can be seen only at time scales greater than the period of the harmonic forcing. The usual way to describe periodic forcing and couple it to fluid dynamics involves perturbation theory ${ }^{16,17}$ and then developing velocity, pressure, and density fields as

$$
\begin{aligned}
& v=v_{0}+\epsilon v_{1}+\epsilon^{2} v_{2}, \\
& p=p_{0}+\epsilon p_{1}+\epsilon^{2} p_{2}, \\
& \rho=\rho_{0}+\epsilon \rho_{1}+\epsilon^{2} \rho_{2},
\end{aligned}
$$

where $\epsilon=k A, k$ being the wavenumber and $A$ being the amplitude of the wave. In this paper, in view of our operating frequency range (between 15 and $120 \mathrm{~Hz}$ ), typical values of the wavelength exceed $10 \mathrm{~m}$, so much higher than any other characteristic length scale involved. The quantity $\epsilon$ consequently remains very small in our experiments, yet it is not always the case in acoustic streaming phenomena, especially at frequencies greater than $1 \mathrm{MHz} .^{8}$

The continuity and Navier-Stokes equations for a compressible Newtonian fluid yields

$$
\begin{aligned}
\partial_{t} \rho+\nabla \cdot(\rho v) & =0, \\
\rho \partial_{t} v+\rho(v \cdot \nabla) v & =-\nabla p+\eta \nabla^{2} v+\frac{1}{3} \eta \nabla(\nabla \cdot v),
\end{aligned}
$$

where bulk viscosity is neglected. Here, we consider an initially unperturbed fluid, $v_{0}=0$. Under the usual adiabatic and barotropic conditions, $p=p(\rho)$ and can be expanded around $\rho_{0}$ as

$$
p=p_{0}+\left(\rho-\rho_{0}\right) c^{2}+\frac{1}{2}\left(\rho-\rho_{0}\right)^{2}\left(\partial_{\rho} c^{2}\right)_{0},
$$

with $c=\left(\partial_{\rho} P\right)_{s}$ the velocity of the sound wave. Injecting Eq. (3) in Eq. (2) and considering only the terms proportional to $\epsilon$ lead to the first-order equations

$$
\begin{aligned}
\partial_{t} \rho_{1} & =-\rho_{0} \nabla \cdot v_{1}, \\
\rho_{0} \partial_{t} v_{1} & =-c^{2} \nabla \rho_{1}+\eta \nabla^{2} v_{1}+\frac{1}{3} \eta \nabla\left(\nabla \cdot v_{1}\right) .
\end{aligned}
$$

Coupling these two equations and neglecting the viscous terms in Eq. (4a) yields to wave equations for $v_{1}, \rho_{1}$, and $p_{1},{ }^{16}$ as expected in classical linear acoustic theory. The second-order equations, however, are more complex since terms in $\epsilon^{2}$ are either linear with respect to a second-order quantity or a product of two first-order ones. Therefore, the Navier-Stokes becomes

$$
\rho_{0} \partial_{t} v_{2}+\rho_{1} \partial_{t} v_{1}+\rho_{0}\left(v_{1} \cdot \nabla\right) v_{1}=-c^{2} \nabla \rho_{2}-\frac{1}{2}\left(\partial_{\rho} c^{2}\right)_{0} \nabla \rho_{1}^{2}+\eta \nabla^{2} v_{2}+\frac{1}{3} \eta \nabla\left(\nabla \cdot v_{2}\right)
$$

Depending in particular on the frequency of the wave, several forms of flows originate from different mechanisms. ${ }^{18}$ The first kind of flow, initially investigated by Rayleigh ${ }^{19}$ and further explained by Schlichting, ${ }^{20}$ is boundary layer-induced regions of non-zero vorticity lie inside the viscous boundary layer around the vibrating object or acoustic transducer, the remaining region being globally irrotational. The outer (Rayleigh) streaming is a consequence of the continuity of stresses between the two regions. Stationary flow patterns, such as those of Tatsuno's work with an oscillating cylinder in a glycerine solution, ${ }^{21}$ can arise and exhibit two regions corresponding to the inner and outer 


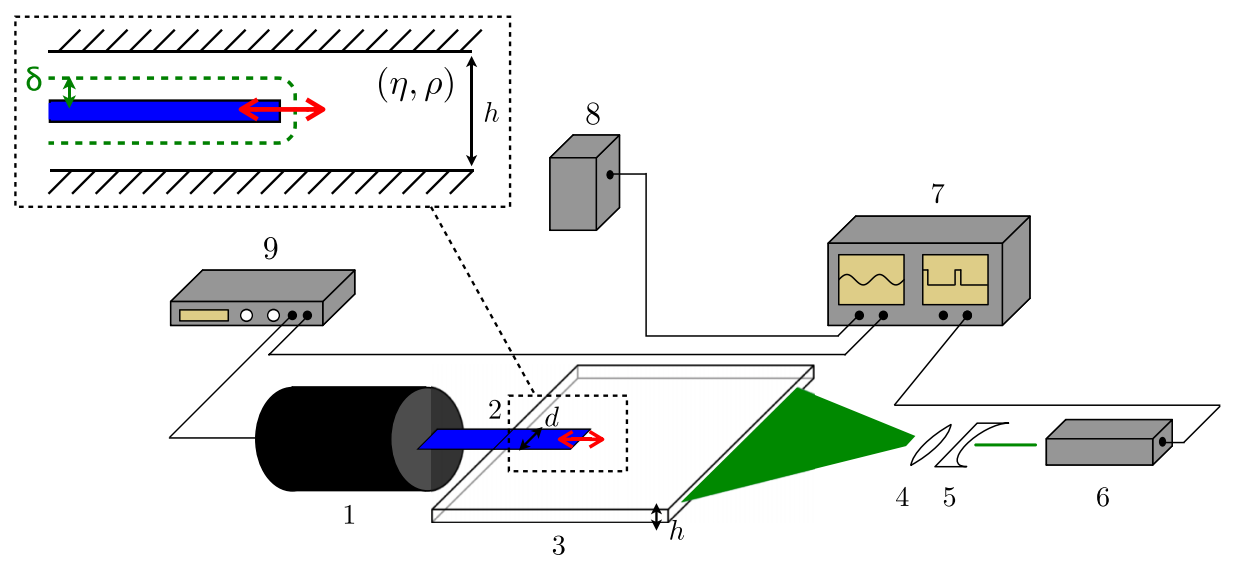

FIG. 1. Sketch of the experimental setup. A function generator (7) prescribes a periodic sinusoidal voltage to an amplifier (9), then is turned to the periodic mechanical forcing of a beam (2) fixed on top of a vibrator (1), and inserted in a Hele-Shaw cell (3) containing the liquid seeded with $10 \mu \mathrm{m}$ silver coated hollow glass spheres (for the flow visualization). The bold arrows (red online) show the vibration direction. An optical set up composed of a cylindrical (5) and a convex (4) lens, and a Nd:Yag laser (6) lights the cell with a 2D sheet of light. Movies are recorded by a camera (8) installed on top.

streaming. At much higher frequencies $(\geq 10 \mathrm{MHz})$, the dimension of the vessel is more likely to be larger than the attenuation length $L_{\text {att }}$ of the sound wave by viscosity. ${ }^{22}$ Therefore, viscous dissipation when the sound wave propagates into the bulk fluid is responsible for the fluid motion, called Eckart streaming. ${ }^{23}$ Recalling that frequency does not exceed $200 \mathrm{~Hz}$ in this paper, only Rayleigh streaming occurs.

Although compressibility can play a significant role in acoustic streaming,${ }^{24}$ this effect is not relevant in the present situation, since the Mach number based on the first-order velocity $v_{1}$ keeps very small

$$
M a=\frac{v_{1}}{c} \sim \frac{A \omega}{c}
$$

where $\omega$ denotes the pulsation of the forcing. Considering maximum values of frequency and amplitude of $120 \mathrm{~Hz}$ and $5 \mathrm{~mm}$, respectively, $M a=2.5 \times 10^{-3}$, so we will neglect every term related to compressibility in Eq. (5). The simplified time-averaged second-order Navier-Stokes equation yields from Eq. (5)

$$
\rho\left\langle\left(v_{1} \cdot \nabla\right) v_{1}\right\rangle=\eta \nabla^{2}\left\langle v_{2}\right\rangle,
$$

where the only non-zero time-averaged term arises from inertia, generating a non-zero second-order steady velocity. In the horizontal $x$ and $y$ direction, i.e., the plane of the cell, the smallest length scale is given by the size of the beam, $d=10 \mathrm{~mm}$, which is much higher than the biggest length scale in the vertical direction, that is to say the Hele-Shaw cell thickness $h=2 \mathrm{~mm}$. The continuity equation $\nabla \cdot v=0$ then implies $v_{z} \sim(h / d) v_{x, y}$, so ensures a two-dimensional flow. Moreover, in the third term of Eq. (7), $\nabla^{2}$ sums up to $\partial^{2} / \partial z^{2}$. Therefore, this viscous term has to be evaluated through a characteristic width $l_{z}$ in which all the stresses are concentrated. Thus, a scaling approach allows us to estimate, from Eq. (7), this steady velocity

$$
\eta \frac{\left\langle v_{2}\right\rangle}{l_{z}^{2}} \sim \rho \frac{\left\langle v_{1}^{2}\right\rangle}{h},
$$

where $v_{1} \sim A \omega$ is the forcing velocity. $l_{z}$ is set equal to the boundary layer thickness $\delta \sim \sqrt{2 v / \omega}$, with $v$ the kinematic viscosity of the fluid, while the thickness of the Hele-Shaw cell remains greater than $\delta$. Equation (8) eventually yields

$$
\left\langle v_{2}\right\rangle \sim \frac{A^{2} \omega}{h} .
$$




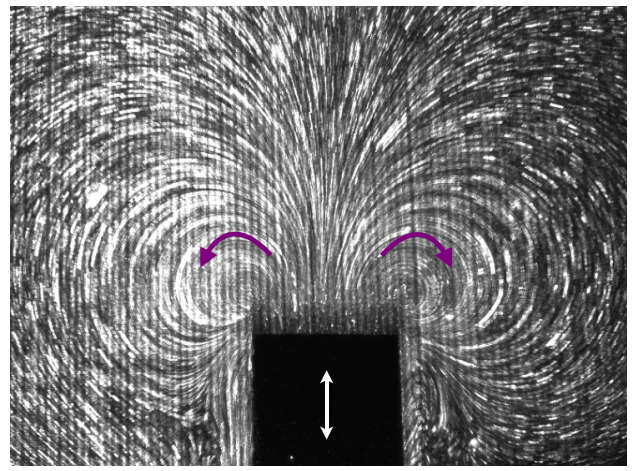

(a)

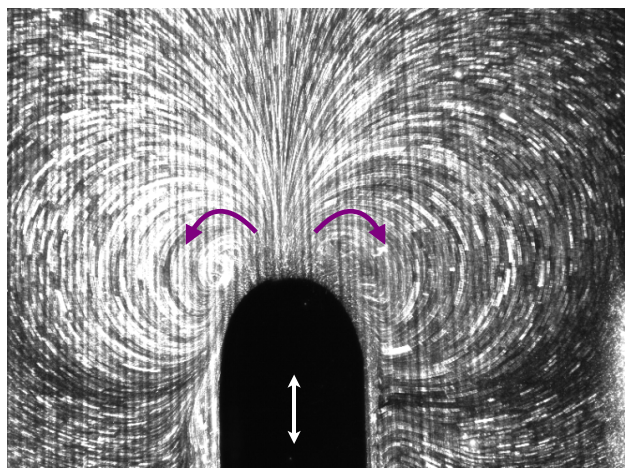

(b)

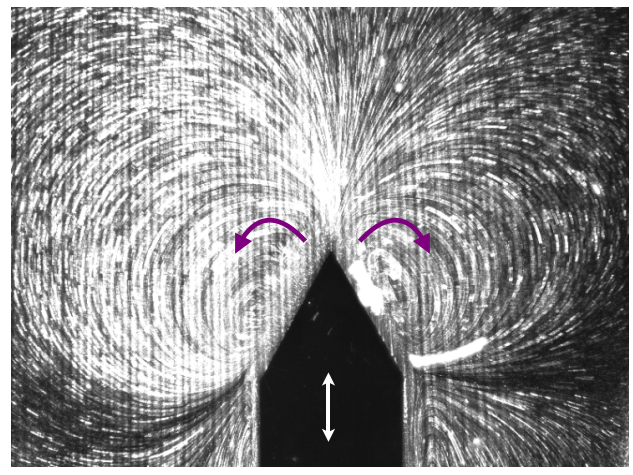

(c)

FIG. 2. Superimposition of frames of flow patterns generated by square 10-mm beams ( $3.7 \mathrm{~s}$ exposure time) (a), rounded 10-mm-diameter beams ( $4.2 \mathrm{~s}$ exposure time) (b), and pointed 10-mm-wide beams (6.0 s exposure time) (c), with $f=50$ $\mathrm{Hz}$ and $A=2.3 \mathrm{~mm}$. White double arrows indicate the direction of vibrations. Bold arrows (purple online) show the rotation direction of the vortices.

The other important underlying property is the independence of the scaling law on viscosity. We have carried out experiments to confirm this, and the domain of validity of this scaling law is discussed further in Sec. V.

\section{EXPERIMENTAL SETUP}

Fig. 1 shows the experimental setup: a Hele-Shaw cell made of two flat glass plates (Fig. 1(3)) $2 \mathrm{~mm}$ apart. This gap is filled with water or water/glycerine solutions in which we insert a metallic beam $10 \mathrm{~mm}$ wide, $1.5 \mathrm{~mm}$ thick, and $100 \mathrm{~mm}$ long (typical immersed length between 40 and $50 \mathrm{~mm}$ ) (Fig. 1(2)). The beam is coated with black paint to avoid light reflections and vibrates along the longitudinal direction in a frequency range from 15 to $120 \mathrm{~Hz}$. Capillary forces acting on the meniscus around the beam and plates prevent leakage from the cell. With our vibration generator (Brüel \& Kjær type 4810) (Fig. 1(1)) and power amplifier (Brüel \& Kjær Power Amplifier type 2718) (Fig. 1(9)), the oscillation amplitude can then be fixed up to $1 \mathrm{~mm}$ with an accuracy better than $0.01 \mathrm{~mm}$ in our frequency range. Since the velocity of sound in water is approximately $1500 \mathrm{~m} / \mathrm{s}$, our working wavelength exceeds $10 \mathrm{~m}$, hence much greater than the cell size. This mechanical forcing can be understood as an analogous to the emission of an acoustic plane wave by the beam of much smaller size than the effective acoustic wavelength.

Flow patterns are observed by particle-image velocimetry (PIV): water is seeded with silvercoated hollow glass spheres of $10 \mu \mathrm{m}$ diameter (Dantec Dynamics), the cell is illuminated by a laser sheet generated by a $4 \mathrm{~W}$ maximum power Nd:Yag laser (Fig. 1(6)) beam crossing a cylindrical lens (Fig. 1(5)) and a convex lens (Fig. 1(4)) to focus it in our region of interest. The whole is filmed from above with a camera (Basler A312f) of $53 \mathrm{fps}$ maximum frame rate (Fig. 1(8)). With this geometry 
and the direction of vibration of the metallic beam, two regions are of interest in this experimental framework: a shear region on both sides of the beam and a compression region in front of it, so flows should arise from a combination of these sources.

\section{RESULTS}

\section{A. Flow patterns}

As the beam starts to vibrate, a stationary flow is generated whose streamlines can be visualized by superimposing several consecutive images. This technique lets us record the trajectories of fluid particles, as seen in Fig. 2. Several kinds of patterns can be observed depending on the frequency and amplitude of the vibration. Starting from a pattern with only two vortices centered near the boundary between the shear and the compression regions at low frequency and amplitude (Fig. 3(a)), the centers of these vortices shift toward the axis of the vibrating beam and are pushed away from it as the frequency or amplitude increases (Fig. 3(b)). Then, the flow can evolve to a quadripolar shape with two vortices on both sides of the plate in the shear boundary area and two others in front of it in the compression area, all of them converging to its corners (Fig. 4(a)). Further increasing frequency and amplitude leads to enlargement of the vortices, which can break into pairs in the shear region (Figs. 3(c) and 3(d)).

To demonstrate that the corners of the square beam are not the origins of vortices, Fig. 2(b) shows a picture of the flow generated by a circular vibrating beam in which the same kind of patterns is clear. The long-time exposure shots in Fig. 2, as well as in Tatsuno ${ }^{21}$ for an oscillating cylinder in an unconfined geometry, are in a good qualitative agreement with those of the Rayleigh Nyborg Westervelt streaming described by Lighthill in Ref. 15 . These type of flows are also observed for acoustically excited bubbles in microchannels, ${ }^{25}$ corroborating that we obtain similar flow patterns for different

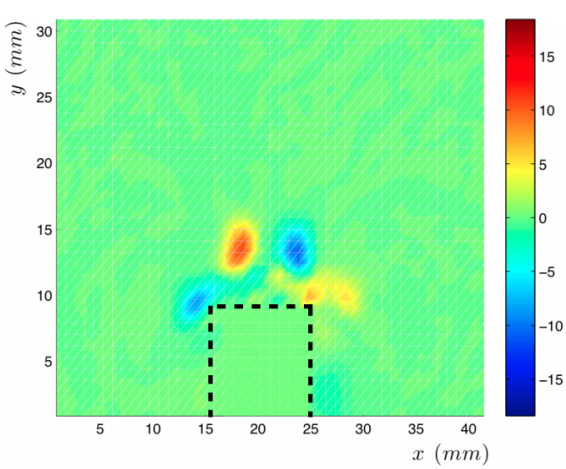

(a)

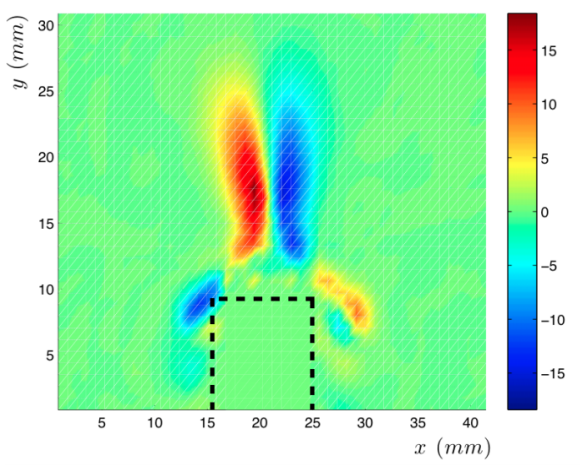

(c)

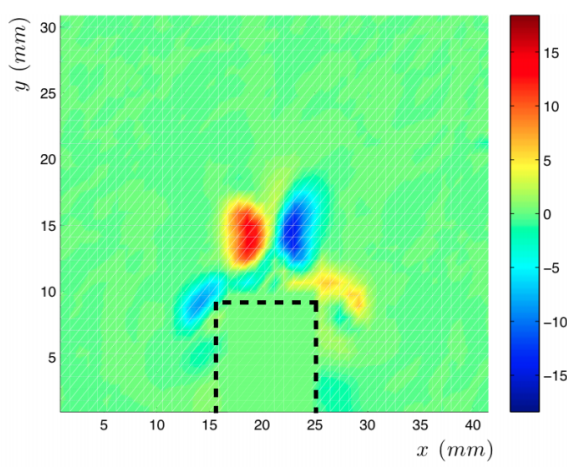

(b)

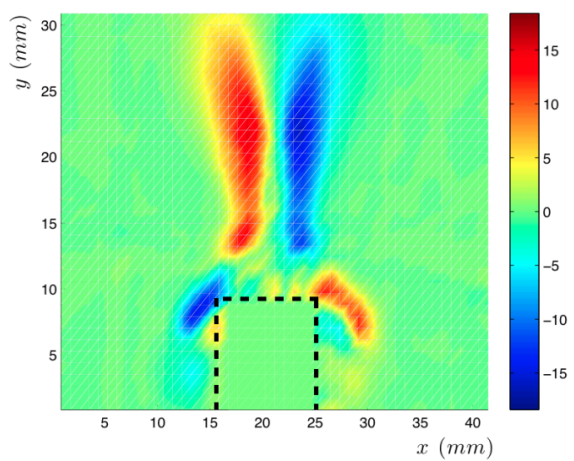

(d)

FIG. 3. Vorticity maps of flow generated in pure water by a vibration of $f=50 \mathrm{~Hz}$ and $A=1.2 \mathrm{~mm}$ (a), $A=1.7 \mathrm{~mm}$ (b), $A=2.1 \mathrm{~mm}(\mathrm{c})$, and $A=2.8 \mathrm{~mm}(\mathrm{~d})$. Dashed lines delimit the vibrating beam. 


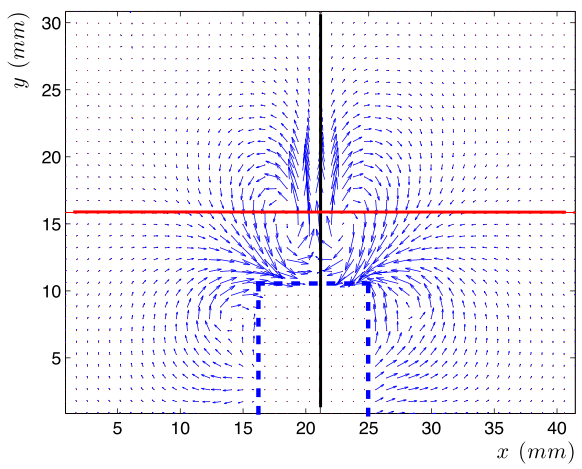

(a)

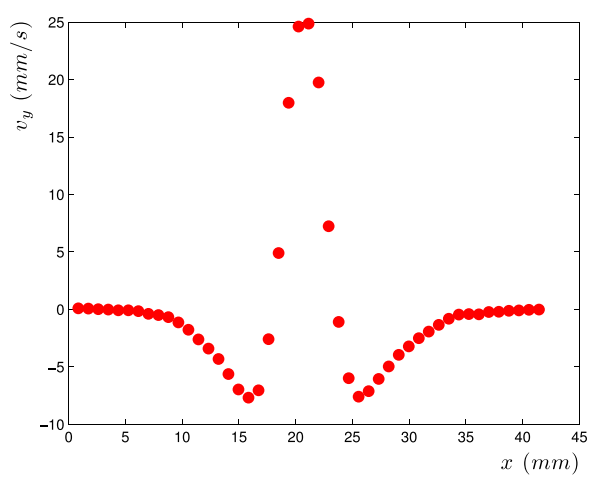

(c)

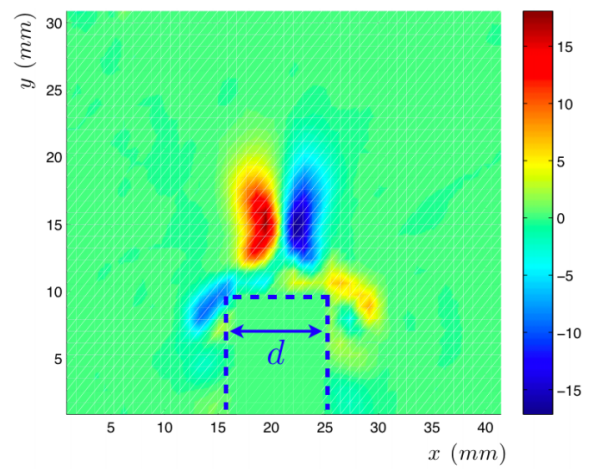

(b)

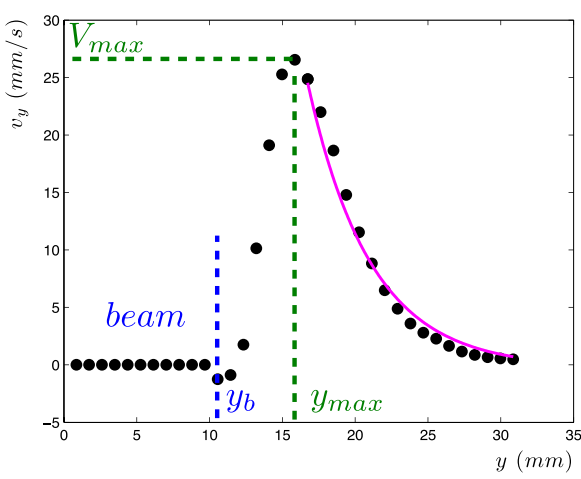

(d)

FIG. 4. Average velocity field based on 441 fields, corresponding to a time of $22.1 \mathrm{~s}$ (a) and vorticity map (b) of the flow generated in the neighborhood of a $f=50 \mathrm{~Hz}, A=1.8 \mathrm{~mm}$ vibrating beam. (c) and (d) are the $x$ and $y$ profiles, respectively. The purple solid line in (d) is a fitting curve in the form $V_{\max } \exp \left(-\left(y-y_{\max }\right) / s\right), V_{\max }$, and $y_{\max }$ being known.

shapes of vibrating object. This suggests that the flow pattern is a robust feature depending largely on the size of the beam and the forcing.

After recording a movie of the flow, we use ImageJ software for image processing such as background subtraction and contrast enhancement. Then, the velocity fields of each pair of successive frames are analyzed with an adaptative correlation code from Dynamic Studio software (Dantec Dynamics). Since the flow remains in a stationary state as long as the vibration continues, it enables to reduce potential errors and consequent data noise through time-averaging of velocity fields to form a clear view of the actual average flow phenomena. An example of such velocity field is shown in Fig. 4(a). The region without velocity vectors corresponds to the mask of the vibrating beam. In order to quantify the influence of the frequency and the amplitude of the excitation, the maximum velocity in the compression area is extracted from each velocity field, and vorticity maps of the flow are calculated.

\section{B. Flow dynamics}

Extraction of the maximum velocity along the axis of the beam (see Fig. 4(d)) from the velocity fields shows a trend suggesting a linear relationship between $V_{\max }$ and the excitation frequency (Fig. 5). This behavior is in good agreement with scaling law (9) established in Sec. II. Comparing the velocities for pure water and for a water-glycerin mixture at $\eta=10.5 \mathrm{cP}$, we observed the same trend. However, the prefactor relating $V_{\max }$ and $f$ clearly depends on viscosity. These results demonstrate that the case of water-glycerin mixture is out of the range of validity of scaling law (9). We come back to this point in Sec. V. 


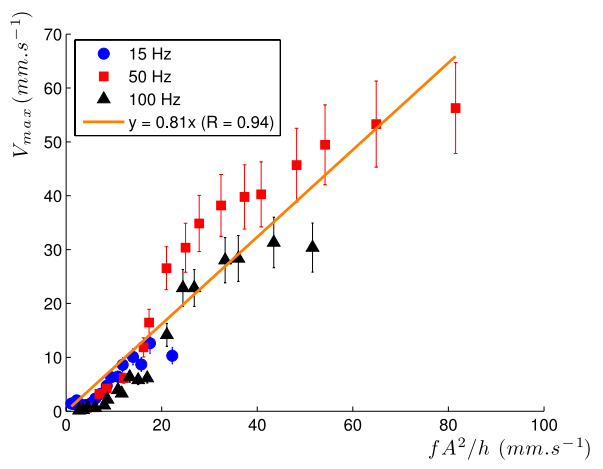

(a)

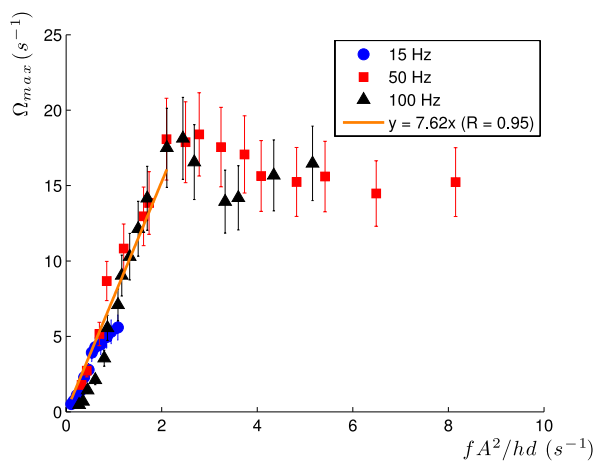

(c)

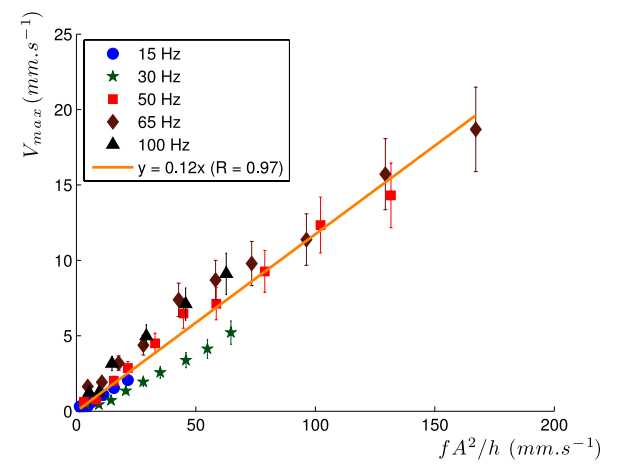

(b)

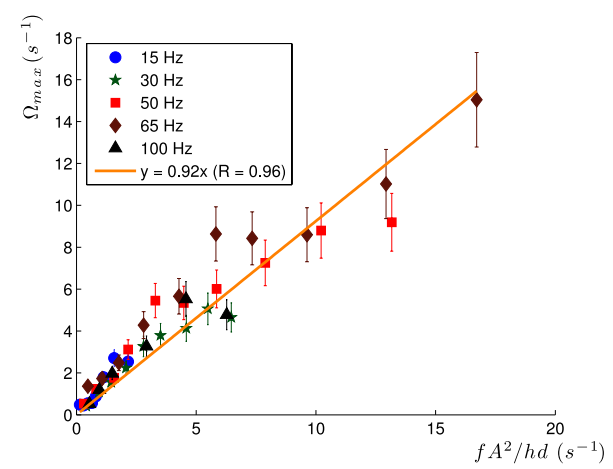

(d)

FIG. 5. Maximum velocity as a function of $\frac{A^{2} f}{h}$ in (a) pure water and (b) water-glycerin mixture $(\eta=10.5 \mathrm{cP})$ and maximum vorticity as a function of $\frac{A^{2} f}{h d}$ in pure water (c) and in a water-glycerine mixture of $\eta=10.5 \mathrm{cP}$ (d).

\section{Vorticity}

The influence of frequency and amplitude on flow vorticity is also investigated. Invoking Stokes' theorem, we expect vorticity $\Omega$ to be related to velocity $v$ by

$$
\int_{(C)} \boldsymbol{v} \cdot \boldsymbol{d l}=\iint_{(S)} \boldsymbol{\Omega} \cdot \boldsymbol{d} \boldsymbol{S} .
$$

Vorticity maps (Fig. 3) emphasize the dependence of vortex shape on excitation amplitude and frequency, so estimating $(S)$ and hence choosing $(C)$ are not straightforward. Vortices are confined in the $x$ direction, as confirmed by flow profiles (see Fig. 4(c)), limited to the vibrating beam width, yet they can stretch in the $y$ direction without geometrical constraints (see Fig. 3(d)), with a typical extension of $l$. Equation (10) thus scales as

$$
v l \sim \Omega l d .
$$

Introducing Eq. (9) and dropping $l$ leads to

$$
\Omega \sim \frac{A^{2}}{h d} \omega .
$$

Hence, vorticity should evolve in the same way as velocity, with $A^{2} \omega$. Measuring maximum (or minimum) vorticity deduced from the velocity fields confirms this prediction experimentally (Figs. 5(c) and 5(d)). However, this vorticity trend based on scaling arguments shows a departure from a purely linear relationship at relatively strong forcing, emphasized for low viscosities: vorticity reaches a plateau as the forcing acceleration increases, as shown in Fig. 5(c), corresponding to the generation of vortices on both sides of the beam (Fig. 3), where vorticity is redistributed. 


\section{DISCUSSION}

The scaling laws for both velocity and vorticity come out of a balance between inertia-generating a steady flow from the periodic forcing-and viscous dissipation confined in the boundary layer around the vibrating beam. This reasoning leads to independence on viscosity, which remains relevant only if $h \ll \delta$. Since $\delta \sim \sqrt{v / \omega}$, a departure from this scaling may occur at low frequencies (see Fig. 5(b)). To illustrate this behavior qualitatively, let us look at Fig. 6, corresponding to experiments performed with a water/glycerin mixture of $\eta=108.2 \mathrm{cP}$.

Increasing $v$ turns the visible flow patterns to a double-layer flow, as noted by Stuart ${ }^{26}$ and Tatsuno, ${ }^{21}$ as inner streaming takes over a larger part of the flow field. Far from the transition between these two flows, the size of this layer is ruled by $\delta$ and $d$ but is independent on $A$, so at a given viscosity, reducing the excitation frequency makes this layer more visible. As shown in Fig. 6, the flow generated by a $200 \mathrm{~Hz}$ vibration (Fig. 6(d)) exhibits two vortices, forming a jet away from the beam. At $120 \mathrm{~Hz}$ (Fig. 6(c)), the inner streaming appears, formed by a pair of vortices directed towards the beam and confined in a layer in front of it; the size of this layer increases as the frequency is reduced (see Fig. 6(b)) until it expands to the whole cell at $10 \mathrm{~Hz}$ (Fig. 6(a)). Unlike for the outer streaming, increasing the amplitude does not lead to a displacement of the inner vortices.

Furthermore, the transition from this double to a single layer flow structure, corresponding to the fast shrinkage of the inner layer as the frequency increases, seems to be influenced by the confinement provided by the Hele-Shaw configuration. The gap $h$ of the latter is likely to act as a cutoff length for the vibrating beam size $d$, since the boundary layer thickness $\delta$, growing around the vibrating beam (see Fig. 1), reaches the same order of magnitude. However, since $d$ still affects the size of the outer vortices and hence flow expansion, it should be of interest in applications such as mixing or transfer enhancement in confined geometries.

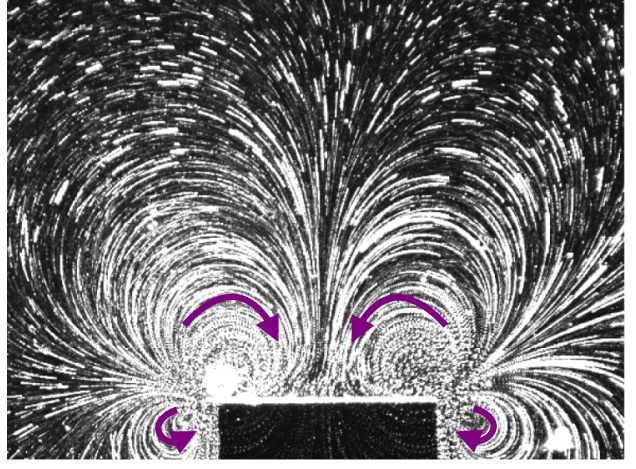

(a)

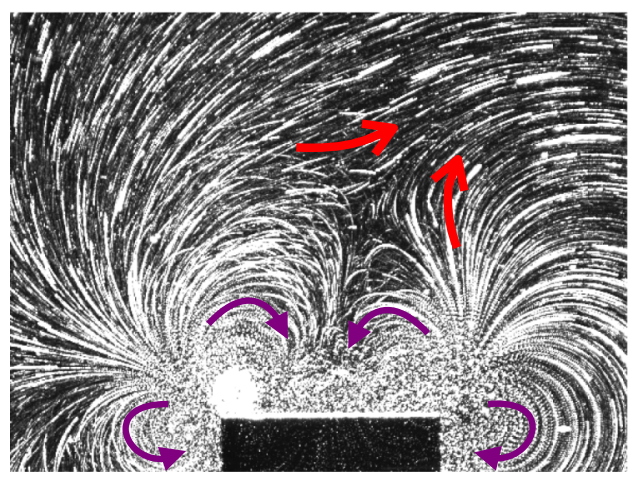

(c)

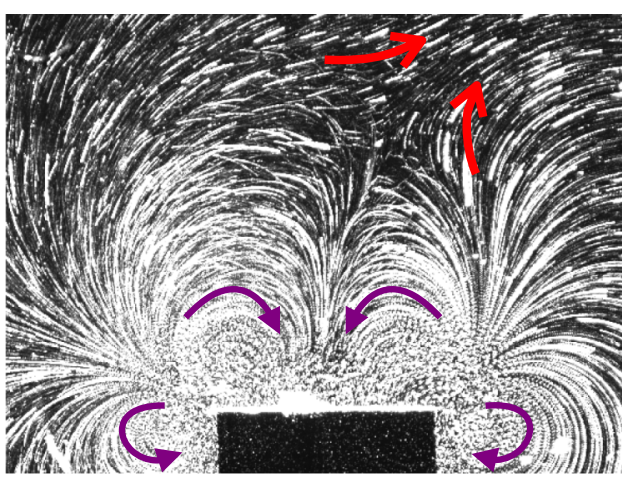

(b)

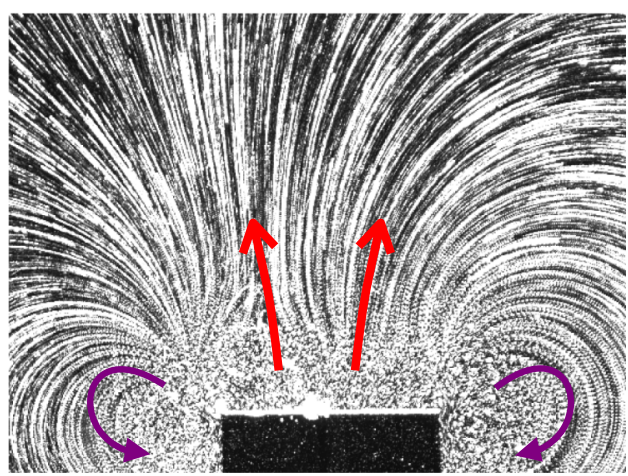

(d)

FIG. 6. Evidence of the inner (rotational) streaming flow with a higher-viscosity liquid : superimposition of frames of flows generated by a $d=10.0 \mathrm{~mm}$-width beam in water-glycerin mixture of $\eta=108.2 \mathrm{cP}$ vibrating at $A=0.4 \mathrm{~mm}$ and $f=50 \mathrm{~Hz}$ (a), $f=100 \mathrm{~Hz}$ (b), $f=120 \mathrm{~Hz}$ (c), and $f=200 \mathrm{~Hz}$ (d), corresponding exposure time $150 \mathrm{~s}$. Thin (purple) and bold (red) arrows indicate the rotation direction of the vortices in the inner and outer streaming, respectively. 
This expansion, regarded along the axis of vibration (black solid line in Fig. 4(a)), can be decomposed into two parts: the distance between the end of the beam $y_{b}$ and the position $y_{\max }$ of the maximum streaming velocity $V_{\max }$ (see Fig. 4(d)) on the one hand, and the attenuation length $s$ of the velocity from $y_{\max }$ to the infinity (Hele-Shaw cell dimensions) on the other. As the amplitude and the frequency increase, the position of the maximum velocity $V_{\max }$ shifts away from the beam. The distance $y_{\max }-y_{b}$ consequently follows the $f A^{2}$ trend given by scaling law Eq. (9) with no influence of $d$. Further than $y_{\max }$, the streaming velocity along the axis of vibration decreases with a characteristic attenuation length $s$. Assuming an exponential decay, $s$ is evaluated by a fit of the form (purple curve on Fig. 4(d))

$$
v_{y}(y)=V_{\max } \exp \left(-\left(y-y_{\max }\right) / s\right)
$$

recalling that $y_{\max }$ and $V_{\max }$ are known. As shown in Fig. 7, whatever the amplitude and frequency of the excitation, $s$ is comparable to $d / 2$, half of the vibrating beam width

A similar statement emerges from measuring the amplitudes $A_{1}$ of the flow harmonic response, evidenced by oscillations of the seeding particles during one period, in the vicinity (dashed blue line in Fig. 7(a)) of the axis of vibration of the beam (dashed purple line in Fig. 7(a)). A plot of $A_{1}$, normalized by its maximum value $A_{1, m}$, versus the distance $y-y_{b}$ to the beam (Fig. 7(b)) suggests an exponential decay. Following the same approach conducted to evaluated $s$, a characteristic attenuation length $l_{a}$ can be obtained by fitting this average amplitude with $A_{1, \text { off }} / A_{1, m}+\exp \left(-\left(y-y_{b}\right) / l_{a}\right)$ (red curve in

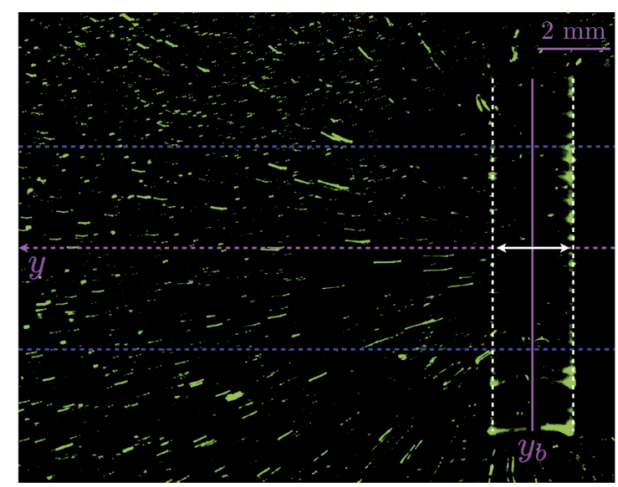

(a)

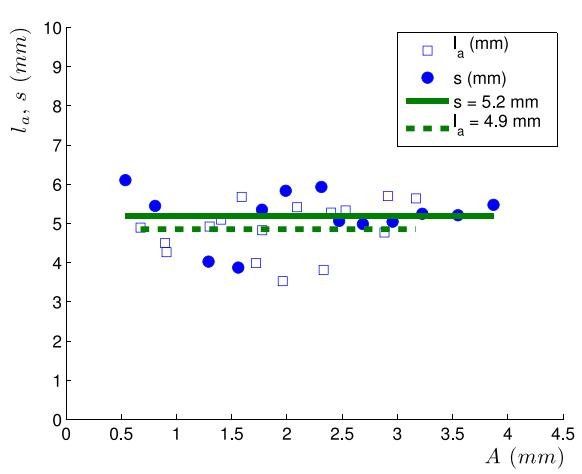

(c)

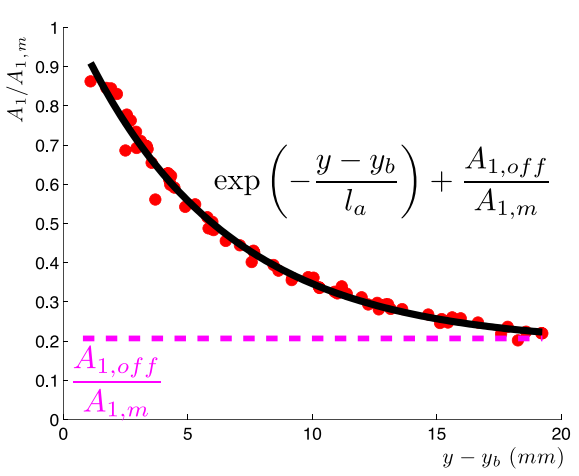

(b)

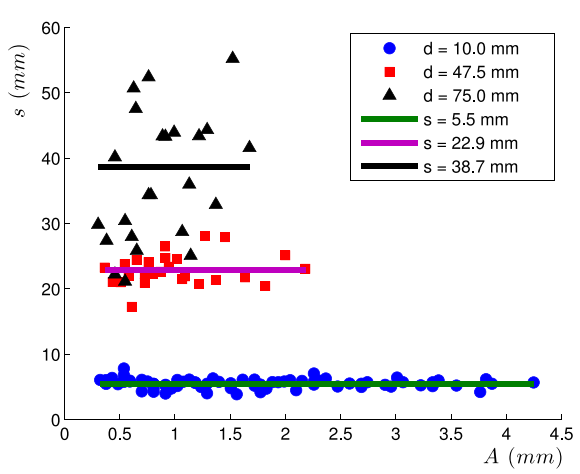

(d)

FIG. 7. The flow generated in a glycerin mixture $(\eta=10.5 \mathrm{cP})$ by a $10 \mathrm{~mm}$-width vibrating beam at $f=50 \mathrm{~Hz}$ and $A=2.3 \mathrm{~mm}$ (a). The white arrow indicates the vibration direction and the amplitude is delimited by white dashed line. The region in which measurements were done is indicated by the two dashed blue lines around the axis of vibration (dashed purple line). (b) The corresponding plot of measured amplitude $A_{1} / A_{1, \mathrm{~m}}$ of the fluid harmonic response as a function of the distance to the beam $y-y_{b}$, from which we can extract an attenuation length $l_{a}$ fitted by an exponential decrease plus an offset: $\exp \left(-\left(y-y_{b}\right) / l_{a}\right)+A_{1, \mathrm{off}} / A_{1, \mathrm{~m}}$ (solid black line). (c) The attenuation length $l_{a}$ from the fit of (b) and characteristic length of decay $s$ extracted from velocity profiles along the axis of vibration as a function of $A$, the continuous and dashed lines corresponding to the average values. (d) The decay length $s$ as a function of $A$ for several frequencies and beam widths $d$, with continuous lines showing the average values. 
Fig. 7(b)), where $A_{1, \mathrm{~m}}$ is the maximal amplitude measured and $A_{1, \mathrm{off}}$ a fitting parameter corresponding to an offset on $A_{1}$. As for the slope $s$ emanating from the velocity $y$-profile (Fig. 4(d)), the evaluation of $l_{a}$ always returns a length close to $d / 2$, whatever amplitude or frequency of the excitation within our working range. In summary, the characteristic length of the decrease $s$ of the second-order flow obtained from Fig. 4(d) is comparable to that extracted from the first-order oscillations of the flow in Fig. 7(b). Since this feature is not noticed in extended geometries, it could be attributed to the two-dimensional confinement of our geometry.

\section{CONCLUSION}

Our study presents quantitative measurements on streaming flows forced by mechanical vibrations within a two-dimensionally confined geometry. We found behaviors which are analogous to acoustically forced streaming flows, because the vibrating object can be considered as a diffuser of an acoustic plane wave of wavelength much larger than the size of the vessel. This is typically the case in ultrasonic wave-forced flows in microfluidics geometries. The results of our PIV measurements yield simple scaling laws for the maximal velocity and vorticity and show the limitations of these laws, especially in capturing the influence of viscosity and vibrating object size. The scaling law is valid typically when the size of the boundary layer is less than other characteristic length of the problem, primarily the thickness of the liquid gap. Therefore, the prescribed geometrical confinement must be taken into account in the flow structure and spatial reach.

Ongoing work concerns viscous and size effects as well as flow dynamics of the inner streaming. The authors are currently working to reduce the scale of their experiments in order to study that kind of streaming at microscale, particularly its efficiency in mixing.

\section{ACKNOWLEDGMENTS}

The authors are grateful to Julien Seznec, Laurent Réa, and Matthieu Receveur for their technical support. We would like to thank Michael Baudoin and Professor Henrik Bruus for fruitful discussions.

${ }^{1}$ M. Faraday, "On a peculiar class of acoustical figures; and on certain forms assumed by groups of particles upon vibrating elastic surfaces," Philos. Trans. R. Soc. London 121, 299-340 (1831).

${ }^{2}$ N. Riley, "Steady streaming," Ann. Rev. Fluid Mech. 33, 43-65 (2001).

${ }^{3}$ C. Suri, K. Takenaka, H. Yanagida, Y. Kojima, and K. Koyama, "Chaotic mixing generated by acoustic streaming," Ultrasonics 40(1-8), 393-396 (2002)

${ }^{4}$ Y. Choe and E. S. Kim, "Valveless micropump driven by acoustic streaming," J. Micromech. Microeng. 23(4), 045005 (2013).

${ }^{5}$ N. P. Dhanalakshmi, R. Nagarajan, N. Sivagaminathan, and B. V. S. S. S. Prasad, "Acoustic enhancement of heat transfer in furnace tubes," Chem. Eng. Process. 59(0), 36-42 (2012).

${ }^{6}$ G. Destgeer, K. H. Lee, J. H. Jung, A. Alazzam, and H. J. Sung, "Continuous separation of particles in a pdms microfluidic channel via travelling surface acoustic waves (tsaw)," Lab Chip 13, 4210-4216 (2013).

${ }^{7}$ H. Kumar, M. H. Tawhai, E. A. Hoffman, and C.-L. Lin, "Steady streaming: A key mixing mechanism in low-Reynoldsnumber acinar flows," Phys. Fluids 23(4), 041902 (2011).

${ }^{8}$ J. Friend and L. Y. Yeo, "Microscale acoustofluidics: Microfluidics driven via acoustics and ultrasonics," Rev. Mod. Phys. 83(2), 647-704 (2011).

${ }^{9}$ X. Ding, P. Li, Sz-Chin Steven Lin, Z. S. Stratton, N. Nama, F. Guo, D. Slotcavage, X. Mao, J. Shi, F. Costanzo, and T. J. Huang, "Surface acoustic wave microfluidics," Lab Chip 13, 3626-3649 (2013).

${ }^{10}$ M. Tatsuno and P. W. Bearman, "A visual study of the flow around an oscillating circular cylinder at low Keulegan Carpenter numbers and low Stokes numbers," J. Fluid Mech. 211(2), 157-182 (1990).

${ }^{11}$ H. Dutsch, F. Durst, S. Becker, and H. Lienhart, "Low-Reynolds-number flow around an oscillating circular cylinder at low Keulegan Carpenter numbers," J. Fluid Mech. 360(4), 249-271 (1998).

12 J. R. Elston, H. M. Blackburn, and J. Sheridan, "The primary and secondary instabilities of flow generated by an oscillating circular cylinder," J. Fluid Mech. 550(3), 359-389 (2006).

${ }^{13}$ C. P. Lee and T. G. Wang, "Outer acoustic streaming," J. Acoust. Soc. Am. 88(5), 2367-2375 (1990).

${ }^{14} \mathrm{H}$. An, L. Cheng, and M. Zhao, "Direct numerical simulation of oscillatory flow around a circular cylinder at low Keulegan-Carpenter number," J. Fluid Mech. 666(1), 77-103 (2011).

15 J. Lighthill, “Acoustic streaming,” J. Sound Vib. 61(3), 391-418 (1978).

${ }^{16}$ H. Bruus, Theoretical Microfluidics (Oxford University Press, 2008).

${ }^{17}$ S. S. Sadhal, "Acoustofluidics 13: Analysis of acoustic streaming by perturbation methods," Lab Chip 12(13), 2292-2300 (2012).

${ }^{18}$ S. Boluriaan and P. Morris, “Acoustic streaming: From Rayleigh to today,” Int. J. Aeroacoustics 2(3), 255-292 (2003). 
${ }^{19}$ L. Rayleigh, "On the circulation of air observed in Kundt's tube, and on some allied acoustical problems," Philos. Trans. R. Soc. London 1776-1886 175, 1-21 (1884).

${ }^{20}$ H. Schlichting, "Calculation of even periodic barrier currents," Phys. Z. 33, 327-335 (1932).

${ }^{21}$ M. Tatsuno, "Circulatory streaming around an oscillating circular cylinder at low Reynolds numbers," J. Phys. Soc. Jpn. 35(3), 915-920 (1973).

${ }^{22}$ M. F. Hamilton and D. T. Blackstock, Nonlinear Acoustics (Academic Press, 1998).

${ }^{23}$ C. Eckart, "Vortices and streams caused by sound waves," Phys. Rev. 73(1), 68-76 (1948).

${ }^{24}$ Q. Qi, "The effect of compressibility on acoustic streaming near a rigid boundary for a plane traveling wave," J. Acoust. Soc. Am. 94(2), 1090-1098 (1993).

${ }^{25}$ C. Wang, B. Rallabandi, and S. Hilgenfeldt, "Frequency dependence and frequency control of microbubble streaming flows," Phys. Fluids 25(2), 022002 (2013).

${ }^{26}$ J. T. Stuart, "Double boundary layers in oscillatory viscous flow," J. Fluid Mech. 24(4), 673-687 (1966). 